\title{
Efficacy of Cardiac Resynchronization Therapy in Patients with a Narrow QRS Complex
}

\author{
Toshiko Nakai (D), Yukitoshi Ikeya, Hiroaki Mano, Rikitake Kogawa, Ryuta Watanabe, \\ Masaru Arai, Yoshihiro Aizawa, Sayaka Kurokawa, Kimie Ohkubo, Daisuke Kitano, \\ Koichi Nagashima, and Yasuo Okumura
}

\author{
Nihon University School of Medicine, Department of Medicine, Division of Cardiology, Tokyo, Japan \\ Correspondence should be addressed to Toshiko Nakai; nakai.toshiko@nihon-u.ac.jp
}

Received 15 September 2020; Revised 5 January 2021; Accepted 12 March 2021; Published 20 March 2021

Academic Editor: Yuichiro Maekawa

Copyright (c) 2021 Toshiko Nakai et al. This is an open access article distributed under the Creative Commons Attribution License, which permits unrestricted use, distribution, and reproduction in any medium, provided the original work is properly cited.

\begin{abstract}
Aims. In the guidelines for cardiac resynchronization therapy (CRT), there is a gap between the Japanese Circulation Society (JCS) criteria, which specify a QRS duration of $\geq 120 \mathrm{~ms}$, and other countries, with a QRS $\geq 130 \mathrm{~ms}$. The efficacy of CRT remains controversial in patients with a narrow QRS $<130 \mathrm{~ms}$. The aims of this study are to evaluate the response to CRT in patients with a narrow QRS and to identify predictors of mortality. Methods. We retrospectively studied 212 patients who received CRT. They were divided into narrow QRS $(<130 \mathrm{~ms})$ and wide QRS $(\geq 130 \mathrm{~ms})$ groups. We compared CRT response rates and investigated whether age, gender, baseline New York Heart Association (NYHA) class, ischemic etiology, atrial fibrillation, and ventricular arrhythmias are associated with response and also predictive of mortality. Results. The CRT response rate was not significantly different between the wide QRS group and the narrow QRS group (74.6\% versus $77.2 \%, p=0.6876$ ), and the response rate in the narrow QRS group was as good as that reported worldwide. NYHA class IV was shown to be a predictor of mortality (HR 9.38, $95 \%$ CI 5.35-16.3, $p<0.0001)$. Conclusions. The present study demonstrated that patients with a narrow QRS complex responded well to CRT. Even with QRS $<130 \mathrm{~ms}$, CRT should be tried if no other effective treatment is available.
\end{abstract}

\section{Introduction}

Despite the fact that cardiac resynchronization therapy (CRT) has been shown to be an effective treatment for heart failure (HF) [1-4], it is clearly underutilized in Japan. According to the Japanese Registry of All Cardiac and Vascular Diseases (JROAD) survey, the number of patients hospitalized for HF in 2017 was 260,000 and is increasing by 10,000 per year [5]. This registry confirmed the data previously recorded in the Chronic Heart Failure Analysis and Registry in the Tohoku District (CHART) study and data from earlier reports $[6,7]$. In contrast, the total number of CRT implantations in combination with either a pacemaker or defibrillator, as reported by the Japan Arrhythmia Device Industry Association (JADIA) in 2017, was about 3300/year [8], and this number has remained fairly static in recent years. While not all HF patients are candidates for CRT, considering the prevalence of interventricular conduction disturbance (IVCD) in HF $[9,10]$, a somewhat larger increase in the use of CRT would have been expected. Differences in the guidelines between Japan and other countries, especially the European Society of Cardiology (ESC) guidelines [11], primarily account for this underutilization. The Japanese Circulation Society (JCS) guidelines recommend CRT for patients whose QRS is $\geq 120 \mathrm{~ms}$ [12], whereas the ESC guidelines classified patients with a narrow QRS complex of $<130 \mathrm{~ms}$ as class III. Thus, the current situation has led to some hesitation when considering CRT implantation in patients with a relatively narrow QRS complex, ranging between $120 \mathrm{~ms}$ and $130 \mathrm{~ms}$. However, in clinical practice, we deal with numerous cases where patients with a narrow QRS complex of $<130 \mathrm{~ms}$ respond well to CRT [13-15]. The aim of this study was to evaluate the CRT response in patients with a narrow QRS complex of $<130 \mathrm{~ms}$ compared with those with wide QRS complex of $\geq 130 \mathrm{~ms}$. 


\section{Methods}

2.1. Subjects. 269 consecutive patients who underwent CRT implantation at Nihon University Itabashi Hospital between April 2010 and March 2020 were retrospectively studied. CRT was performed according to the Japanese Circulation Society Guidelines in patients who fell into classes I, IIa, and IIb indication [12]. Patients who were upgraded to CRT while receiving right-ventricular pacing because they were pacemaker-dependent were excluded because the intent was different from that of this study. A total of 212 patients were analyzed. Patients were divided into a narrow QRS $(<130 \mathrm{~ms}$ [range, 120-128], $n=63)$ group and wide QRS $(\geq 130 \mathrm{~ms}$ [range, 130-238], $n=149$ ) group. We compared CRT response rates between the two groups and performed a multivariable analysis to determine whether QRS duration, LBBB morphology, baseline New York Heart Association (NYHA) functional class IV, ischemic etiology, atrial fibrillation (AF), gender, ventricular arrhythmias, and device type (CRT-P) are predictive of mortality. This study was approved by our institutional ethics committee.

2.2. CRT Programming. AV optimization was performed using automatic optimization, which is incorporated into each device, in order to achieve a narrower QRS compared with the baseline QRS complex. However, if the QRS did not narrow sufficiently with the automatic optimization algorithm, we programmed the AV delay and VV delay manually. We start biventricular pacing with the AV delay $10 \mathrm{~ms}$ less than the intrinsic PR interval and prolong the AV delay in $10 \mathrm{~ms}$ increments while looking at the QRS width on the ECG; we decided to program the AV delay that yielded the narrowest QRS. We did not optimize VV delay in all cases, but if the QRS did not look narrower following AV delay optimization, we adjusted the VV delay by $10 \mathrm{~ms}$ to find the narrowest QRS. CRT reoptimization was performed if the QRS looked wider than the baseline QRS at any clinic visits.

\subsection{Definition of CRT Responder. A "CRT response" was} defined as having occurred provided the patient met criteria for both functional and echocardiographic responses. A functional response at six-month follow-up was defined as an improvement of at least one grade in "functional status" based on the NYHA class. An echocardiographic response was defined as a reduction in the left ventricular end systolic volume of $\geq 15 \%$ or an improvement in the LVEF (left ventricular ejection fraction) of $\geq 5 \%$ at six months after CRT implantation, based on past clinical trials and reports $[1-3,16,17]$. LVEF was measured by the Simpson method.

2.4. Statistical Analysis. Continuous variables are presented as the mean value \pm standard deviation (SD) or standard error (SE). Categorical variables are expressed as total number and percentages. Differences between groups with narrow and wide QRS complex and responder and nonresponder groups were analyzed by $t$-test or chi-square test, as appropriate. Survival was evaluated using the KaplanMeier method. The effect of different variables on survival, including age, gender, QRS duration, non-LBBB morphology, NYHA functional class IV, ischemic etiology, presence of AF, ventricular arrhythmias, and CRT-pacemaker usage, was investigated using the Cox proportional hazards model. Variables that showed a statistically significant effect on survival in univariate analyses were entered in a multivariate Cox proportional hazards model, using backward, stepwise selection to obtain the final model. All statistical analyses were performed with JMP 14.0 software (SAS Institute, Cary, NC, USA), and $p$ values $<0.05$ were considered significant.

\section{Results}

3.1. Subjects. The median follow-up period was 35 months [range, 0-192]. The subjects were 170 men and 42 women with a median age of 66 years [range, 14-90]. Mean QRS duration was $145 \pm 17 \mathrm{~ms}$ with $16 \%$ of patients falling into NYHA functional class IV HF. In 74 patients (34\%), the etiology could be classified as ischemic. A CRT-pacemaker (CRT-P) was implanted in 62 patients (29\%) and a CRTdefibrillator (CRT-D) was implanted in 150 patients.

The baseline characteristics of study patients as well as a comparison between narrow and wide QRS patients are summarized in Table 1. There was no significant difference except QRS duration between the narrow and wide QRS patients.

3.2. CRT Response. As previously described, CRT responder status was determined based on combined assessment of both echocardiographic data and functional status. The echocardiographic data and NYHA functional class obtained before and after CRT implantation as well as the changes in these parameters are shown in Table 2. Reverse remodeling was obtained after CRT implantation in both narrow and wide QRS groups, and none of the parameter changes were significantly different between the two groups. The echocardiographic response rate was $76.4 \%$ (74.6\% in the narrow QRS group and 77.2\% in the wide QRS group); the functional response rate was $85.3 \%$ (84.1\% in the narrow QRS group and $85.9 \%$ in the wide QRS group).

The overall response rate, meeting both criteria, was $76.4 \%$. There was no significant difference in the response rate between the narrow and wide QRS groups (74.6\% versus $77.2 \%$, respectively, $p=0.6876$, Figure 1 ). As shown in Table 3 , in patients with severe $\mathrm{HF}$, there was a significant difference between responder and nonresponder groups $(10 \%$ versus $30 \%, p=0.0005$, respectively). The overall death rate was significantly lower in the responder group compared with the nonresponder group ( $31 \%$ versus $65 \%, p<0.0001$, respectively). Significant predictors of nonresponse were severe HF (NYHA IV) and ischemic etiology. Multivariate analysis revealed that the strongest predictor of nonresponse to CRT was NYHA IV HF (HR 3.43, 1.55-7.64, $p=0.0024$ ). Narrow QRS was not a significant predictor of CRT nonresponse.

3.3. Overall Mortality. During the mean follow-up period of $48 \pm 44$ months, 85 patients died (40\%). Of these deaths, 45 (52\%) were due to cardiac causes and 40 (47\%) were due to 
TABLE 1: Patient characteristics: overall, narrow, and wide QRS groups.

\begin{tabular}{|c|c|c|c|c|}
\hline & Overall $(n=212)$ & Narrow $(n=63)$ & Wide $(n=149)$ & $p$ value \\
\hline Age (years), mean $\pm S D$ & $66 \pm 13$ & $65 \pm 14$ & $67 \pm 12$ & 0.2291 \\
\hline Sex, male & $171(81 \%)$ & $54(86 \%)$ & $109(79 \%)$ & 0.2152 \\
\hline QRS (ms), mean \pm SD & $145 \pm 17$ & $122 \pm 9$ & $159 \pm 20$ & $<0.0001$ \\
\hline LBBB morphology & $140(66 \%)$ & $38(60 \%)$ & $102(68 \%)$ & 0.2560 \\
\hline NYHA class IV & $33(16 \%)$ & $13(21 \%)$ & $20(13 \%)$ & 0.1949 \\
\hline Ischemic etiology & $83(39 \%)$ & $25(40 \%)$ & $58(39 \%)$ & 0.9179 \\
\hline $\mathrm{AF}$ & $38(18 \%)$ & $11(17 \%)$ & $27(18 \%)$ & 0.9086 \\
\hline VT/VE history & $76(36 \%)$ & $20(33 \%)$ & $56(38 \%)$ & 0.5095 \\
\hline CRT-P (\%) & $62(29 \%)$ & $14(22 \%)$ & $48(32 \%)$ & 0.1373 \\
\hline $\operatorname{LVEF}(\%)$, mean $\pm \mathrm{SD}$ & $28.2 \pm 9.1$ & $28.2 \pm 9.5$ & $28.2 \pm 9.0$ & 0.9921 \\
\hline \multicolumn{5}{|l|}{ Medication } \\
\hline Beta-blocker agent & $189(91 \%)$ & $58(95 \%)$ & $131(90 \%)$ & 0.1899 \\
\hline ACEI/ARB & $138(67 \%)$ & $40(66 \%)$ & $98(67 \%)$ & 0.8296 \\
\hline Diuretics & $185(89 \%)$ & $56(92 \%)$ & $129(88 \%)$ & 0.4531 \\
\hline Overall death & $84(39 \%)$ & $30(48 \%)$ & $54(36 \%)$ & 0.1235 \\
\hline
\end{tabular}

NYHA: New York Heart Association class; AF: atrial fibrillation; VT/VF: ventricular tachycardia/ventricular fibrillation; CRT-P: cardiac resynchronization pacemaker; LVEF: left ventricular ejection fraction; ACEI: angiotensin-converting enzyme inhibitor; ARB: angiotensin receptor blocker; SD: standard deviation; HF: heart failure.

TABLE 2: Echocardiographic data and NYHA functional class at baseline and 6 months after CRT implantation.

\begin{tabular}{|c|c|c|c|c|c|c|c|}
\hline & \multicolumn{3}{|c|}{ Narrow QRS group } & \multicolumn{3}{|c|}{ Wide QRS group } & \multirow[b]{2}{*}{$p$ value ${ }^{*}$} \\
\hline & $\begin{array}{c}\text { Baseline } \\
(\text { mean } \pm \mathrm{SD})\end{array}$ & $\begin{array}{c}6 \text { months } \\
(\text { mean } \pm S D)\end{array}$ & $\begin{array}{c}\text { Delta } \\
(\text { mean } \pm S D)\end{array}$ & $\begin{array}{c}\text { Baseline } \\
(\text { mean } \pm \mathrm{SD})\end{array}$ & $\begin{array}{c}6 \text { months } \\
(\text { mean } \pm \mathrm{SD})\end{array}$ & $\begin{array}{c}\text { Delta } \\
(\text { mean } \pm S D)\end{array}$ & \\
\hline LVEF (\%) & $28.2 \pm 9.5$ & $36.1 \pm 9.7$ & $7.9 \pm 9.8$ & $28.2 \pm 9.0$ & $34.2 \pm 11.0$ & $5.9 \pm 10.2$ & 0.2768 \\
\hline LVEDV (mL) & $223 \pm 69$ & $204 \pm 65$ & $16 \pm 53$ & $214 \pm 64$ & $186 \pm 75$ & $27 \pm 50$ & 0.2584 \\
\hline LVESV (mL) & $162 \pm 65$ & $134 \pm 56$ & $25 \pm 50$ & $154 \pm 56$ & $127 \pm 63$ & $26 \pm 43$ & 0.9012 \\
\hline NYHA class & $3.1 \pm 0.6$ & $2.0 \pm 0.8$ & $1.0 \pm 0.6$ & $3.0 \pm 0.5$ & $1.9 \pm 0.8$ & $1.1 \pm 0.6$ & 0.6453 \\
\hline
\end{tabular}

LVEF: left ventricular ejection fraction; LVEDV: left ventricular end diastolic volume; LVESV: left ventricular end systolic volume; "Delta" indicates the difference before and after implantation; SD: standard deviation. ${ }^{*} P$ value difference in delta between narrow and wide QRS groups.

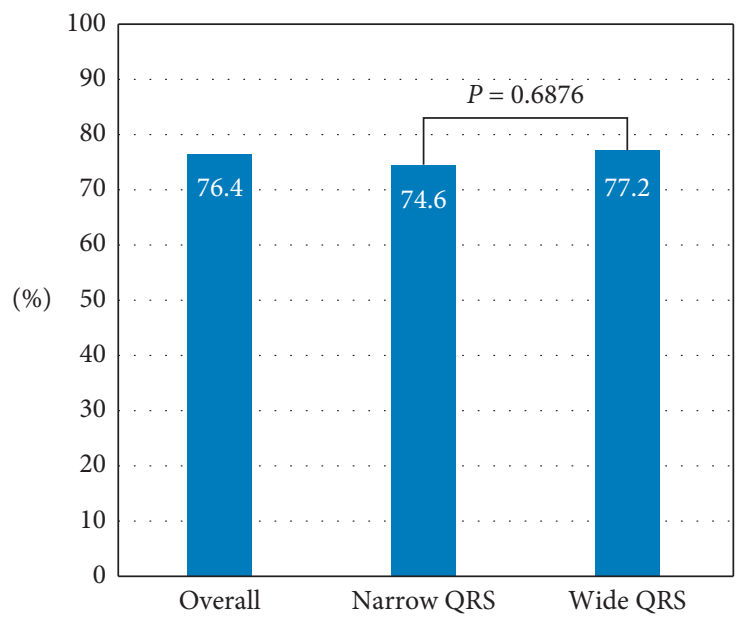

FIGURE 1: CRT response rate, overall, narrow, and wide QRS groups.

noncardiac causes; in nine patients, the etiology of death could not be determined and was classified as unknown. Overall survival based on QRS duration and NYHA class is shown in Figure 2. After 150 months of follow-up, there was no significant survival difference between the narrow and wide QRS groups $(p=0.2361)$. Of the patients with NYHA class IV HF, just 37\% (12/33) were still alive 12 months after CRT implantation. There was a significant difference in 
TABLE 3: Patient characteristics: responders versus nonresponders and predictors of nonresponse to CRT.

\begin{tabular}{|c|c|c|c|c|c|c|c|}
\hline & \multirow{2}{*}{$\begin{array}{c}\text { Responder } \\
(n=155)\end{array}$} & \multirow{2}{*}{$\begin{array}{c}\text { Nonresponder } \\
\quad(n=57)\end{array}$} & \multirow{2}{*}{$p$ value } & \multicolumn{4}{|c|}{ Predictors of nonresponse } \\
\hline & & & & Univariate OR $(95 \% \mathrm{CI})$ & $P$ Value & Multivariate OR (95\% CI) & $p$ value \\
\hline Age (years), mean \pm SD & $66 \pm 12$ & $67 \pm 13$ & 0.7668 & $1.00(0.98-1.03)$ & 0.7646 & & \\
\hline Sex, male & $121(78 \%)$ & $50(88 \%)$ & 0.1146 & $2.01(0.88-5.20)$ & 0.1016 & & \\
\hline $\mathrm{QRS}(\mathrm{ms})$, mean $\pm \mathrm{SD}$ & $146 \pm 27$ & $144 \pm 23$ & 0.5763 & $1.00(0.99-1.01)$ & 0.5732 & & \\
\hline Narrow QRS $(<130 \mathrm{~ms})$ & $47(30 \%)$ & $16(28 \%)$ & 0.7504 & $0.90(0.45-1.73)$ & 0.7495 & $0.80(0.39-1.60)$ & 0.5396 \\
\hline LBBB morphology & $102(66 \%)$ & $38(67 \%)$ & 0.9066 & $0.73(0.36-1.54)$ & 0.4077 & & \\
\hline NYHA class IV & $16(10 \%)$ & $17(30 \%)$ & 0.0005 & $3.69(1.71-8.03)$ & 0.0010 & $3.43(1.55-7.64)$ & 0.0024 \\
\hline Ischemic etiology & $52(34 \%)$ & $31(54 \%)$ & 0.0059 & $2.36(1.28-4.41)$ & 0.0063 & $2.14(1.13-4.06)$ & 0.0195 \\
\hline $\mathrm{AF}$ & $25(16 \%)$ & $13(22 \%)$ & 0.2610 & $1.54(0.70-3.22)$ & 0.2703 & & \\
\hline VT/VF history & $52(34 \%)$ & $24(43 \%)$ & 0.2254 & $1.47(0.78-2.75)$ & 0.2286 & & \\
\hline CRT-P (\%) & $46(30 \%)$ & $16(28 \%)$ & 0.8196 & $0.92(0.46-1.78)$ & 0.8191 & & \\
\hline $\operatorname{LVEF}(\%)$ & $28 \pm 9.5$ & $28 \pm 8.2$ & 0.9772 & $1.00(0.96-1.04)$ & 0.9770 & & \\
\hline \multicolumn{8}{|l|}{ Medication } \\
\hline Beta-blocker agent & $143(91 \%)$ & $46(85 \%)$ & 0.0634 & $0.40(0.90-6.68)$ & 0.0774 & & \\
\hline ACE inhibitor/AEB & $107(70 \%)$ & $31(57 \%)$ & 0.0932 & $0.58(0.31-1.11)$ & 0.0971 & & \\
\hline Diuretics & $136(89 \%)$ & $49(91 \%)$ & 0.7042 & $1.23(0.46-3.88)$ & 0.7005 & & \\
\hline Overall death & $48(31 \%)$ & $37(65 \%)$ & $<0.0001$ & & & & \\
\hline
\end{tabular}

Abbreviations as in Table 1. OR: odds ratio; CI: confidence interval.

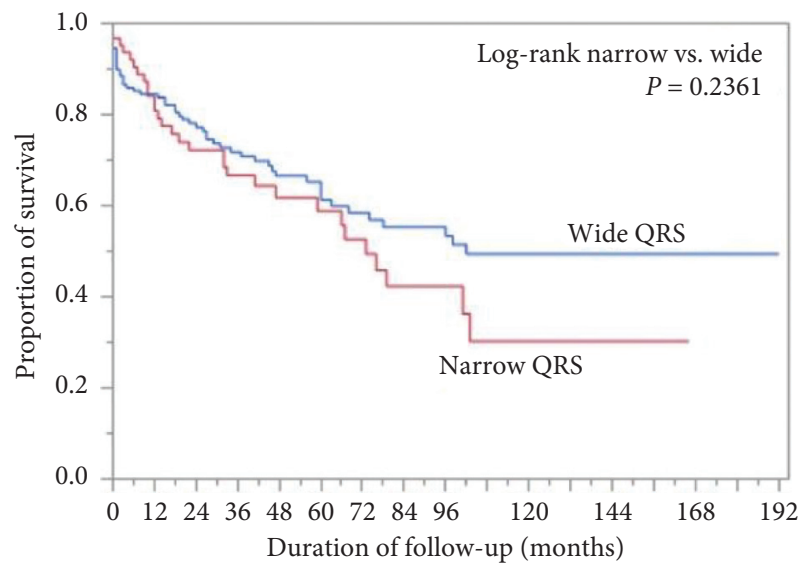

Number at risk

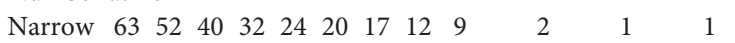

Wide $\quad \begin{array}{llllllllllll}149113 & 91 & 74 & 61 & 49 & 38 & 32 & 29 & 14 & 7 & 4 & 2\end{array}$

(a)

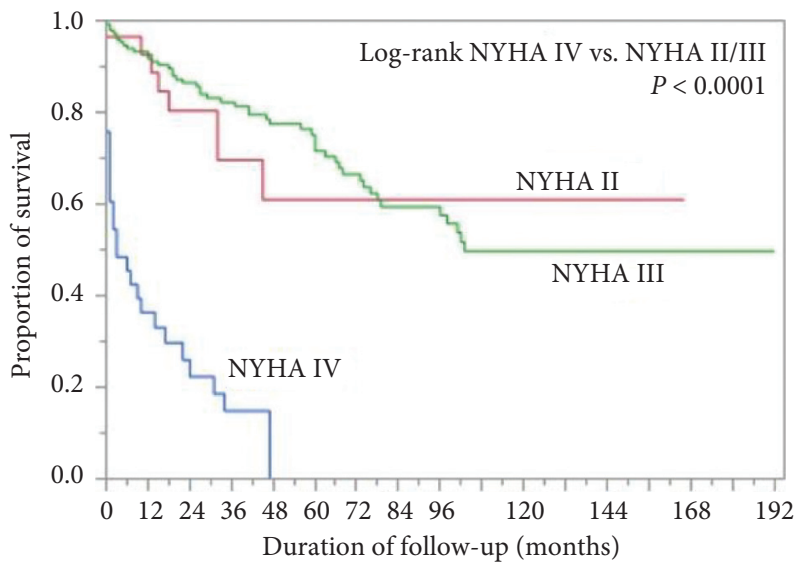

Number at risk

$\begin{array}{lllllll}\text { NYHA IV } & 33 & 12 & 7 & 2 & 1 & 1\end{array}$

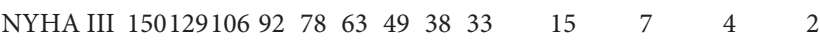
NYHA II $29 \begin{array}{llllllll}24 & 18 & 12 & 7 & 6 & 6 & 6 & 5\end{array}$

(b)

FIGURE 2: (a) Survival curves for narrow and wide QRS groups. There is no significant difference between the two groups. (b) Overall survival based on severity of heart failure severity. (a) Survival curves: wide QRS versus narrow ORS group. (b) Overall survival based on severity of heart failure at baseline.

prognosis between NYHA class IV and NYHA classes II/III $(p<0.0001)$.

Figure 3 shows the outcome based on QRS. There is no significant difference between narrow and wide QRS groups in HF hospitalization and cardiac death $(p=0.4125$ and $p=0.9758$, respectively).

3.4. Predictors of Mortality. Predictors of mortality in all patients are listed in Table 4. Significant univariate predictors of mortality were baseline NYHA class IV (HR 9.25, 95\% CI 5.51-15.4, $p<0.0001$ ), AF (HR 1.78, 95\% CI
1.05-2.89, $p=0.0328)$, absent beta-blocker use (HR 2.18, 95\% CI 1.13-3.88, $p=0.0228)$, and absent ACE inhibitor/ ARB use (HR 1.82, 95\% CI 1.16-2.82, $p=0.0091$ ). After adjusting for these variables in a Cox regression model, the strongest predictor of mortality was baseline NYHA functional class IV (HR 9.38, 95\% CI 5.35-16.3, $p<0.0001$ ).

\section{Discussion}

4.1. Major Findings. CRT response rate did not differ significantly between patients with narrow and wide QRS. Outcomes consisting of $\mathrm{HF}$ hospitalization and cardiac 

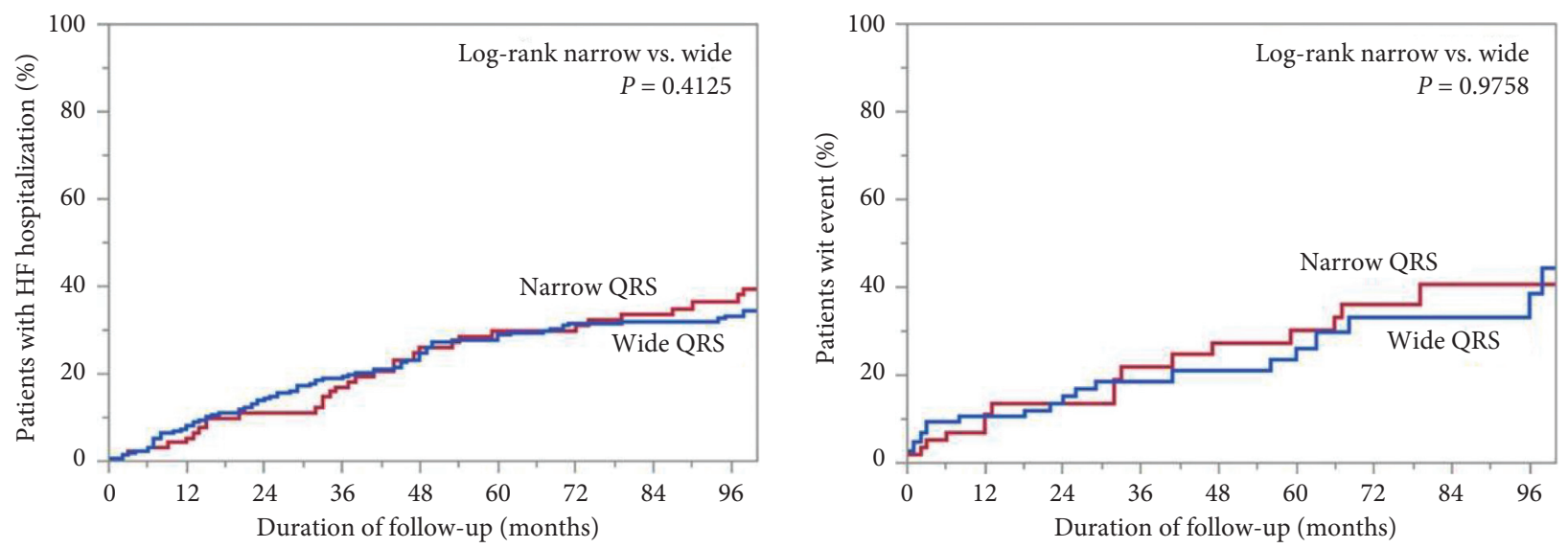

$\begin{array}{lccccccccc}\text { Number at risk } & & & & & & & \\ \text { Narrow } & 63 & 42 & 33 & 27 & 20 & 16 & 16 & 12 & 9 \\ \text { Wide } & 149 & 106 & 84 & 66 & 56 & 44 & 35 & 32 & 29\end{array}$

(a)
Number at risk

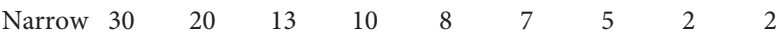

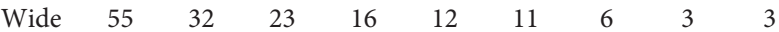

FIGURE 3: Heart failure hospitalization and cardiac death based on QRS duration. (a) HF hospitalization. (b) Cardiac death.

TABLE 4: Predictors of all-mortality risk and univariate and multivariate Cox proportional hazards models.

\begin{tabular}{lccc}
\hline & Univariate HR (95\% CI) & $p$ value & Multivariate HR (95\% CI) \\
\hline Age & $1.00(0.99-1.02)$ & 0.6417 & \\
Sex, male & $1.10(0.65-2.00)$ & 0.7247 & \\
QRS duration & $0.99(0.99-1.01)$ & 0.1542 & $0.84(0.51-1.37)$ \\
Narrow QRS $(<130 \mathrm{~ms})$ & $1.27(0.81-1.97)$ & 0.1333 & \\
LBBB morphology & $1.43(0.90-2.35)$ & $<0.0001$ & $9.38(5.35-16.3)$ \\
NYHA class IV & $9.25(5.51-15.4)$ & 0.0952 & \\
Ischemic etiology & $1.45(0.94-2.22)$ & 0.0328 & $1.66(0.97-2.72)$ \\
AF & $1.78(1.05-2.89)$ & 0.2627 & \\
VT/VF history & $1.29(0.82-2.01)$ & 0.4435 & $1.97(0.99-3.62)$ \\
CRT-P & $1.19(0.75-1.84)$ & 0.0228 & $1.37(0.85-2.16)$ \\
No beta-blocker & $2.18(1.13-3.88)$ & 0.0091 & 0.0655 \\
No ACEI/ARB & $1.82(1.16-2.82)$ & 0.8443 & 0.0544 \\
Diuretic use & $1.07(0.57-2.30)$ & 0.1907 \\
\hline
\end{tabular}

Abbreviations as in Table 1. HR: hazard ratio; CI: confidence interval.

death were also not significantly different between patients with narrow and wide QRS.

4.2. CRT in Patients with a Narrow QRS Complex. Several previous studies indicate that CRT was not effective in patients with a narrow QRS complex, and CRT is now regarded by many as being highly effective only in patients whose QRS is $>150 \mathrm{~ms}$ [1-4]. Moreover, it was concluded that CRT is harmful in patients with a narrow QRS of $<130 \mathrm{~ms}[18,19]$. However, some published studies report a positive CRT response in patients with a narrow QRS complex. They describe the efficacy of CRT, as well as the existence of dyssynchrony in patients with a narrow QRS complex [14, 20-22]. Varma et al. reported efficacy in patients with a narrow QRS complex $<130 \mathrm{~ms}$ from the EchoCRT trial and concluded that CRT may be beneficial in patients with HF with a narrow QRS and smaller ventricles [23]. Indeed, in clinical practice, we often see patients who respond well to CRT despite the fact that their QRS is relatively narrow at $<130 \mathrm{~ms}[15,24]$. In this study, the narrow QRS group had a CRT response rate of 74.6\%, which is an acceptable outcome and on a par with that recorded worldwide $[1-4,16]$.

4.3. AV Optimization in Narrow QRS Patients. It is known that AV optimization is very important for effective CRT. Mullens et al. investigated factors that contribute to CRT nonresponse and found that AV timing is the most frequent reason [25]. We also previously reported favorable outcomes where a nonresponder became a responder after optimization of AV timing [15]. In the cited case, it was challenging to maintain a narrow QRS because the QRS was fairly narrow at baseline. However, we believed that the patient had a conduction disturbance even though the QRS was relatively narrow (less than $130 \mathrm{~ms}$ ), as shown by the presence of left axis deviation. After trial and error, we finally succeeded. Following this experience, we were convinced that CRT might be used successfully in patients with a narrow QRS. 
4.4. Predictors of CRT Nonresponse and Mortality. Most clinical studies show that patients with very severe HF, classified as NYHA class IV, do not respond to CRT and that severity of $\mathrm{HF}$ is an independent predictor of CRT nonresponse $[1-4,26]$. Our findings agree with those of previous reports that NYHA functional class IV is an independent predictor of nonresponse in both narrow and wide QRS groups. Khatib et al. reported the EAARN (ejection fraction (EF), age, AF, renal dysfunction, and NYHA class IV) score is predictive of mortality in CRT patients [24]. They showed that the strongest predictor of mortality was NYHA class IV HF (HR 2.42, 95\% CI $1.62-3.60, p<0.001)$. Our study also showed that the strongest predictor of mortality was baseline NYHA class IV (HR 9.38, 95\% CI 5.35-16.3, $p<0.0001$ ). Kaplan-Meier analysis showed that the survival rate 12 months after CRT implantation is just $38 \%$ in patients with NYHA class IV HR (Figure 2(b)). These data suggest the importance of implantation of a CRT device at an early stage of HF.

As our data show, HF is a progressive disease and early introduction of therapy is the most effective treatment strategy. In this respect, we regard $\mathrm{HF}$ as being very similar to malignant diseases like cancer. It is too late to treat either condition after significant progression. Medication is essential for the treatment of HF, but CRT should be also performed as early as possible if the patient meets the guidelines.

$\mathrm{AF}$ was one of the strongest predictors of mortality (HR $1.78,95 \%$ CI 1.05-2.89, $p=0.0328$ ); however, after multivariate analysis, this became nonsignificant. The response to CRT might be influenced by the percentage of CRT pacing in patients with AF. We endeavored to maintain a higher pacing rate (over 85\%) in CRT patients as part of our CRT management strategy. However, one of the limitations of this study is that we could not ensure an effective pacing rate in all cases, and a pacing rate sufficient to have a clinical effect might not be obtainable in patients with AF.

4.5. Clinical Implications. Our data suggest that patients with a narrow QRS of $<130 \mathrm{~ms}$ can be expected to respond to CRT in a manner similar to patients with a wide QRS complex. However, the response to CRT differs on a case-bycase basis, but withholding CRT just because the QRS is $<130 \mathrm{~ms}$ does not seem rational based on the clinical evidence.

4.6. Study Limitations. Our study has several limitations. First, the number of patients with a narrow QRS complex and the number with a wide QRS complex were far from equal, and this may have affected the statistical analysis. Second, the number of patients with NYHA class IV HF and an ischemic etiology for their HF was limited. This may not accord with the distribution seen globally. However, we believe that the distribution of the etiology of HF in Japan corresponds closely to the distribution in our study patients and can provide some useful information in the selection of candidates for CRT. Third, on comparing responder and nonresponder groups, patients with AF were more often nonresponders, and AF remained a predictor of mortality in the wide QRS group; however, the CRT pacing rate is not reported; thus, whether CRT nonresponse was due to an insufficient pacing rate or due to AF itself is unclear.

\section{Conclusions}

The present study confirms a response to CRT in patients with a narrow QRS complex. Independent predictors of CRT nonresponse and mortality are severe HF in conjunction with NYHA class IV HF. Even patients with a narrow QRS complex of $<130 \mathrm{~ms}$ had a response rate of $>75 \%$, which is on a par with the rate reported worldwide. These results support the idea that the current JCS guidelines are appropriate, at least for Japanese patients, and possibly also for patients with relatively small body size. If heart failure cannot be controlled with medical treatment, CRT should be considered, even in patients with a narrow QRS complex.

\section{Data Availability}

The data used to support the findings of this study are available from the corresponding author upon request.

\section{Ethical Approval}

This study was approved by Nihon University Itabashi Hospital, Clinical Research Judging Committee, Reference number: RK-160510-02.

\section{Disclosure}

Toshiko Nakai belongs to a department established by contributions from Abbot Medical, Biotronik Japan, Medtronic Japan, Japan Lifeline, and Boston Scientific. Toshiko Nakai received lecture fees from Abbot Medical and Medtronic Japan.

\section{Conflicts of Interest}

None of the other authors have conflicts of interest to report.

\section{Authors' Contributions}

Toshiko Nakai, Yukitoshi Ikeya, and Yasuo Okumura designed and drafted the manuscript. Rikitake Kogawa, Yoshihiro Aizawa, Ryuta Watanabe, Masaru Arai, and Sayaka Kurokawa acquired data and performed data analysis. Kimie Ohkubo, Daisuke Kitano, and Koichi Nagashima supported the statistical analysis. Toshiko Nakai and Yasuo Okumura substantially contributed to the manuscript and revised it critically for important intellectual content. All authors approved the submitted version.

\section{References}

[1] W. T. Abraham, W. G. Fisher, A. L. Smith et al., "Cardiac resynchronization in chronic heart failure," New England Journal of Medicine, vol. 346, no. 24, pp. 1845-1853, 2002. 
[2] M. R. Bristow, L. A. Saxon, J. Boehmer et al., "Cardiacresynchronization Therapy with or without an Implantable defibrillator in advanced chronic heart," New England Journal of Medicine, vol. 350, no. 21, pp. 2140-2150, 2004.

[3] J. G. F. Cleland, J.-C. Daubert, E. Erdmann et al., "The effect of cardiac resynchronization on morbidity and mortality in heart failure," New England Journal of Medicine, vol. 352, no. 15, pp. 1539-1549, 2005.

[4] A. S. L. Tang, G. A. Wells, M. Talajic et al., "Cardiacresynchronization therapy for mild-to-moderate heart failure," New England Journal of Medicine, vol. 363, no. 25, pp. 2385-2395, 2010.

[5] "The Japanese registry of all cardiac and vascular diseases (JROAD): annual report 2017," 2019, http://www.j-circ.or.jp/ jittai_chosa/jittai_chosa2016web.pdf.

[6] Y. Okura, M. M. Ramadan, Y. Ohno et al., "Impending epidemic," Circulation Journal, vol. 72, no. 3, pp. 489-491, 2008.

[7] N. Shiba, K. Nochioka, M. Miura, H. Kohno, and H. Shimokawa, "CHART-2 investigators. trend of westernization of etiology and clinical characteristics of heart failure patients in Japan," Circulation Journal, vol. 75, no. 4, pp. 823-833, 2011.

[8] CRT Market Research 2017, "Japan arrhythmia device industry association (JADIA)," 2020, https://www.jadia.or.jp/ date/2017_crtp_eng.pdfhttps://www.jadia.or.jp/date/2017_ crtd_eng.pdf.

[9] K. D. Aaronson, J. S. Schwartz, T.-M. Chen, K.-L. Wong, J. E. Goin, and D. M. Mancini, "Development and prospective validation of a clinical index to predict survival in ambulatory patients referred for cardiac transplant evaluation," Circulation, vol. 95, no. 12, pp. 2660-2667, 1997.

[10] D. Farwell, N. R. Patel, A. Hall, S. Ralph, and A. N. Sulke, "How many people with heart failure are appropriate for biventricular resynchronization?" European Heart Journal, vol. 21, no. 15, pp. 1246-1250, 2000.

[11] P. Ponikowski, A. A. Voors, S. D. Anker et al., "2016 ESC guidelines for the diagnosis and treatment of acute and chronic heart failure: the task force for the diagnosis and treatment of acute and chronic heart failure of the European society of cardiology (ESC). developed with the special contribution of the heart failure association (HFA) of the EFC," European Heart Journal, vol. 37, no. 27, pp. 2129-2200, 2016.

[12] H. Tsutsui, M. Isobe, H. Ito et al., "JCS 2017/JHFS 2017 guideline on diagnosis and treatment of acute and chronic heart failure-digest version," Circulation Journal, vol. 83, no. 10, pp. 2084-2184, 2019.

[13] G. B. Bleeker, M. J. Schalij, S. G. Molhoek et al., "Frequency of left ventricular dyssynchrony in patients with heart failure and a narrow QRS complex," The American Journal of Cardiology, vol. 95, no. 1, pp. 140-142, 2005.

[14] G. B. Bleeker, E. R. Holman, P. Steendijk et al., "Cardiac resynchronization therapy in patients with a narrow QRS complex," Journal of the American College of Cardiology, vol. 48, no. 11, pp. 2243-2250, 2006.

[15] R. Kogawa, T. Nakai, Y. Ikeya et al., "Dramatic response to cardiac resynchronization therapy with AV delay optimization in narrow QRS heart failure," International Heart Journal, vol. 56, no. 6, pp. 671-675, 2015.

[16] E. S. Chung, A. R. Leon, L. Tavazzi et al., "Results of the predictors of response to CRT (PROSPECT) trial," Circulation, vol. 117, no. 20, pp. 2608-2616, 2008.

[17] B. K. Fornwalt, W. W. Sprague, P. BeDell et al., "Agreement is poor among current criteria used to define response to cardiac resynchronization therapy," Circulation, vol. 121, no. 18, pp. 1985-1991, 2010.

[18] F. Ruschitzka, W. T. Abraham, J. P. Singh et al., "Cardiacresynchronization therapy in heart failure with a narrow QRS complex," New England Journal of Medicine, vol. 369, no. 15, pp. 1395-1405, 2013.

[19] R. M. Shah, D. Patel, J. Molnar, K. A. Ellenbogen, and J. N. Koneru, "Cardiac-resynchronization therapy in patients with systolic heart failure and QRS interval $\leq 130 \mathrm{~ms}$ : insights from a meta-analysis," Europace, vol. 17, no. 2, pp. 267-273, 2015.

[20] C.-M. Yu, H. Lin, Q. Zhang, and J. E. Sanderson, "High prevalence of left ventricular systolic and diastolic asynchrony in patients with congestive heart failure and normal QRS duration," Heart, vol. 89, no. 1, pp. 54-60, 2003.

[21] R. J. Van Bommel, H. Tanaka, V. Delgado et al., "Association of intraventricular mechanical dyssynchrony with response to cardiac resynchronization therapy in heart failure patients with a narrow QRS complex," European Heart Journal, vol. 31, no. 24, pp. 3054-3062, 2010.

[22] T. Nakai, H. Mano, Y. Ikeya et al., "Narrower QRS may be enough to respond to cardiac resynchronization therapy in lightweight patients," Heart and Vessels, vol. 35, no. 6, pp. 835-841, 2019.

[23] N. Varma, P. Sogaard, J. J. Bax et al., "Interaction of left ventricular size and sex on outcome of cardiac resynchronization therapy among patients with a narrow QRS duration in the EchoCRT trial," Journal of the American Heart Association, vol. 7, Article ID e009592, 2018.

[24] K. Saito, K. Ibuki, N. Yoshimura et al., "Successful cardiac resynchronization therapy in a 3-year-old girl with isolated left ventricular non-compaction and narrow QRS complex," Circulation Journal, vol. 73, no. 11, pp. 2173-2177, 2009.

[25] W. Mullens, R. A. Grimm, T. Verga et al., "Insights from a cardiac resynchronization optimization clinic as part of a heart failure disease management program," Journal of the American College of Cardiology, vol. 53, no. 9, pp. 765-773, 2009.

[26] M. Khatib, J. M. Tolosana, E. Trucco et al., "EAARN score, a predictive score for mortality in patients receiving cardiac resynchronization therapy based on pre-implantation risk factors," European Journal of Heart Failure, vol. 16, no. 7, pp. 802-809, 2014. 